\title{
Avaliação da protamina na neutralização da heparina após circulação extracorpórea
}

\author{
Roberto Cardoso BARROSO*, José Teles de MENDONÇA*, Marcos Ramos CARVALHO*, \\ Rika Kakuda COSTA*, José Edivaldo dos SANTOS*
}

Barroso R C, Mendonça J T, Carvalho M R, Costa R K, Santos J E - Avaliação da protamina na neutralização da heparina após circulação extracorpórea. Rev Bras Cir Cardiovasc 2002; 17(1): 54-60.

RESUMO: Introdução: A protamina é o antídoto universalmente usado para neutralizar a heparina no final da circulação extracorpórea; porém não existe até o momento consenso sobre uma dose ideal necessária.

Objetivo: Avaliar a efetividade da neutralização da heparina, com doses variadas de protamina após circulação extracorpórea, com três protocolos diferentes.

Casuística e Métodos: Foram randomizados prospectivamente 45 pacientes, no período de abril a agosto de 2000, e divididos em três protocolos: I, II e III, com 15 pacientes cada. O protocolo I avalia a neutralização da heparina, com dose de protamina calculada pela dose inicial de heparina. O protocolo II avalia a neutralização da dose total de heparina usada durante a circulação extracorpórea. O protocolo III avalia a neutralização da dose total de heparina mais um reforço de $30 \%$ dessa dose, administrada em veia periférica durante 4 horas.

Resultados: No protocolo I, $60 \%$ dos pacientes tiveram necessidade de reforço da dose de protamina e $20 \%$ apresentaram complicações hemorrágicas, necessitando de reoperação. Desses $20 \%$, um paciente foi a óbito e outro apresentou acidente vascular cerebral; $53 \%$ dos pacientes do protocolo II tiveram necessidade de reforço da dose de protamina. Os pacientes dos protocolos II e III não apresentaram complicações hemorrágicas nem reoperações.

Conclusões: 1- A dose de protamina para neutralizar a heparina na proporção menor que 1:1 não é suficiente. Os pacientes necessitam de doses de reforço, apresentam mais complicações hemorrágicas e maior necessidade de transfusões. 2 - A neutralização da heparina com dose de protamina na proporção de $1: 1$ não é totalmente eficaz; $53 \%$ dos pacientes necessitaram de doses complementares nas primeiras horas do pós-operatório. 3 - Uma dose adicional de 30\% da dose de protamina na proporção de 1:1 em infusão contínua nas primeiras horas do pós-operatório reduz a perda sangüínea e a necessidade de transfusões.

DESCRITORES: Protaminas, administração e dosagem. Heparina, efeitos adversos. Circulação extracorpórea.

Trabalho realizado na Fundação de Beneficência Hospital Cirurgia e Hospital São Lucas. Aracaju, SE, Brasil. Recebido para publicação em março de 2001.

* Dos Serviços de Cirurgia Cardíaca da Fundação de Beneficência Hospital de Cirurgia e Hospital São Lucas. Endereço para correspondência: Roberto Cardoso Barroso. Rua Frei Luiz Canolo de Noronha, №42 Bairro Ponto Novo. Aracaju, SE, Brasil CEP: 49097-270. Tel. (79) 212-7213 Fax: (79) 217-1241. e-mail: rcbarroso@uol.com.br. 
Barroso R C, Mendonça J T, Carvalho M R, Costa R K, Santos J E - Avaliação da protamina na neutralização da heparina após circulação extracorpórea. Rev Bras Cir Cardiovasc 2002; 17(1): 54-60.

\section{INTRODUÇÃO}

Um dos avanços mais importantes para o desenvolvimento da cirurgia cardíaca com circulação extracorpórea (CEC) foi a descoberta da heparina.

A heparina não fracionada não é uma substância pura e, sim, uma mistura de frações de polissacarídeos de alto e baixo peso molecular, que variam de 3.000 a 40.000 Daltons e cuja funcionalidade em inibir a atividade da trombina (fator Ila) difere de acordo com o peso molecular ${ }^{(1-3)}$.

A heparina é o anticoagulante rotineiramente usado durante a CEC, por ter a vantagem de ser bastante específico, não produzir alergias ou anafilaxia, sendo o efeito colateral mais importante a trombocitopenia (1-3), raramente fatal, que pode ocorrer após 5 a 7 dias de uso continuado da droga $(1,3)$; e existir um antídoto, a protamina, capaz de neutralizar seu efeito (1-3).

Estima-se que cerca de 10 a $20 \%$ dos pacientes submetidos à CEC apresentam sangramento excessivo no pós-operatório imediato, e 5 a $10 \%$ necessitam de re-exploração cirúrgica nas primeiras horas do pós-operatório, devido a sangramento persistente, tamponamento ou acúmulo de coágulos no mediastino ${ }^{(1,4)}$. O sangramento excessivo no pósoperatório pode ser conseqüência de hemostasia cirúrgica deficiente, alterações da coagulação, falta ou excesso de heparina $(1,2,5)$.

A anticoagulação sistêmica para CEC é obtida com dose inicial de heparina de 3 a $4 \mathrm{mg} / \mathrm{kg}$ (300 a $400 \mathrm{Ul} / \mathrm{kg}$ ) e mantida pela administração de doses suplementares de 0,5 a $1 \mathrm{mg} / \mathrm{kg}$ (50 a $100 \mathrm{UI} / \mathrm{kg}$ ) a cada hora de perfusão, conforme as necessidades individuais de cada paciente, mais dose de $25 \mathrm{mg} /$ I de perfusato cristalóide. Alguns Serviços de cirurgia cardíaca usam doses menores (2 mg / kg ), outros, usam doses maiores (5 mg/kg) (1).

É aceito pela maioria dos Serviços que uma anticoagulação adequada durante a CEC deve elevar o tempo de coagulação (TC) em 3 a 4 vezes o seu valor basal; desse modo, seriam indicados valores de tempo de coagulação ativado (TCA) igual ou superior a 480 segundos (1).

Devido a variações no metabolismo e eliminação da heparina, recomenda-se um TCA mínimo de 400 a 480 segundos, representando adequada margem de segurança entre o mínimo absoluto e um excesso desnecessário (6).

O efeito das doses elevadas de heparina ainda é controverso, embora tenha sido demonstrado ativação da coagulação mesmo com TCA superior a 400 segundos $(7,8)$.

A adequada e rápida reversão da heparina é essencial para o controle do sangramento no final da CEC e pós-operatório imediato. O antídoto rotineiramente utilizado para neutralizar o efeito anticoagulante da heparina é o sulfato de protamina.

Devido às reações indesejáveis da protamina ( Tipo $\mathrm{I}=$ hipotensão, $\mathrm{II}=$ anafilactoide e $\mathrm{III}=$ vasoconstricção pulmonar) (3), a droga deve ser administrada diluída e lentamente, sob monitorização contínua, não tendo sido, até o momento, demonstrado qualquer superioridade da administração por via aórtica ou atrial esquerda, sobre via atrial direita ou via venosa clássica $(3,9)$.

A protamina pode provocar efeito hemorrágico em altas doses sangüíneas (1-2 $\mathrm{mg} / \mathrm{ml})$ por reduzir os níveis de fibrinogênio, elevando o tempo de tromboplastina parcial (TTPa) para valores acima de 200 segundos. A dose de protamina necessária para produzir um aumento mínimo do TTPa é de aproximadamente 100 vezes a utilizada para neutralizar a heparina na proporção de $1: 1^{(10,11)}$.

A protamina deve ser usada na dose mínima capaz de neutralizar o efeito anticoagulante de resíduo de heparina na circulação do paciente ao término da CEC, minimizando assim a trombocitopenia transitória (12).

Como o efeito neutralizador da protamina em relação à heparina se faz molécula a molécula, ele varia em função da pureza de ambas as drogas e da meia vida mais curta da protamina (3).

Usa-se, na prática, calcular a neutralização da heparina com protamina, na proporção de 1:1 podendo chegar a 1:1,3 e sendo desnecessário doses acima da proporção de 1:1,5 (1,13-15).

A dose de protamina pode ser utilizada por vários métodos : I - Dose fixa, II - Titulação pelo TCA e III - Determinação da concentração de heparina circulante; porém os resultados ainda não demostram qualquer superioridade entre os métodos $(4,16)$.

A administração lenta de um acréscimo de cerca de $20 \%$ da dose de protamina, nas primeiras 2 ou 3 horas do pós-operatório, pode contribuir para evitar o efeito rebote da heparina e reduzir o volume de sangramento no pós-operatório imediato $(1,15)$.

Alguns pacientes submetidos a CEC e com neutralização da heparina com protamina apresentam ressurgimento da atividade anticoagulante (antitrombínica) após a adequada neutralização com protamina num tempo que variou de 30 minutos a 18 horas (15).

As heparinas são solúveis em água e têm volume de distribuição parecido com o volume plasmático. São metabolizadas pelo fígado, possu- 
Barroso R C, Mendonça J T, Carvalho M R, Costa R K, Santos J E - Avaliação da protamina na neutralização da heparina após circulação extracorpórea. Rev Bras Cir Cardiovasc 2002; 17(1): 54-60.

indo meia - vida entre 90 minutos e 2 horas, sendo afetada por alterações do fluxo sangüíneo hepático, metabolismo e hipotermia $\left(26^{\circ} \mathrm{C}\right.$ a $\left.28^{\circ} \mathrm{C}\right)$, que detêm essencialmente o "clearance" de heparina, permitindo sua liberação tardia após o reaquecimento (3)

A protamina possui meia-vida mais curta, entre 30 e 60 minutos, sendo a diferença entre a meia vida da heparina em relação à da protamina a responsável, parcialmente, pelo fenômeno de rebote ${ }^{(3)}$.

O objetivo deste trabalho foi avaliar a efetividade da neutralização do efeito anticoagulante da heparina, com doses variadas de protamina após a CEC, através de três protocolos diferentes.

\section{CASUÍSTICA E MÉTODOS}

Foi realizado, na Fundação de Beneficência Hospital Cirurgia e Hospital São Lucas, Aracaju SE, um trabalho prospectivo e randomizado, comparando-se 45 pacientes consecutivos, no período de abril a agosto de 2000. Os grupos foram denominados Protocolo I, Protocolo II e Protocolo III, com 15 pacientes em cada grupo.

No protocolo I a idade variou de 11 a 61 anos, com média de $51,6 \pm 16,8$ anos, o peso variou de 40 a $104 \mathrm{~kg}$ com média de 70,9 $\pm 15,9 \mathrm{~kg}$, o tempo de circulação extracorpórea variou de 65 a 150min com média de $101 \pm 24,5 \mathrm{~min}$, sendo 6 pacientes do sexo feminino e 9 do sexo masculino.

No protocolo II a idade variou de 13 a 73 anos, com média de 41,3 $\pm 20,8$ anos, o peso variou de 35 a $85 \mathrm{~kg}$ com média de $59 \pm 15,3 \mathrm{~kg}$, o tempo de circulação extracorpórea variou de 35 a $165 \mathrm{~min}$, com média de $103 \pm 32,8 \mathrm{~min}$, sendo 5 pacientes do sexo feminino e 10 do sexo masculino.

No protocolo III a idade variou de 11 a 67 anos, com média de 44,2 $\pm 19,4$ anos, o peso variou de 34,5 a $80 \mathrm{~kg}$ com média de $55,4 \pm 12,8 \mathrm{~kg}$, o tempo de circulação extracorpórea variou de 40 a $140 \mathrm{~min}$, com média de $88,6 \pm 26,2 \mathrm{~min}$, sendo 5 pacientes do sexo feminino e 10 do sexo masculino.

Foram excluídos do trabalho todos os pacientes que apresentavam coagulopatia prévia, uso de anticoagulante, antiagregante plaquetário, trombolítico e doenças que interferissem na coagulação.

Na sala de operação foi realizado o tempo de coagulação basal, em seguida administrada heparina na dose fixa e padronizada de $5 \mathrm{mg} / \mathrm{kg}$ (dose inicial) mais $1 \mathrm{mg} / \mathrm{kg}$ a cada 40 minutos de CEC (dose de reforço), $25 \mathrm{mg}$ no prime cristalóide, e $50 \mathrm{mg}$ para cada $500 \mathrm{ml}$ de unidade de sangue ou plasma utilizado durante a CEC quando necessário uso de colóides no prime.
O protocolo I rege a neutralização do efeito anticoagulante da dose fixa de $5 \mathrm{mg} / \mathrm{Kg}$ de heparina inicialmente administrada no átrio direito mais os $25 \mathrm{mg}$ de heparina usados no prime cristalóide antes da CEC.

O protocolo II rege a neutralização do efeito anticoagulante da dose total de heparina usada durante a CEC. A dose total foi calculada pelo somatório das doses: inicial usada no átrio direito $(5 \mathrm{mg} / \mathrm{kg})$ mais dose do prime $(25 \mathrm{mg})$, mais as doses de reforço utilizadas durante a CEC (1 mg/kg a cada $40 \mathrm{~min}$ de CEC).

O protocolo III rege a neutralização do efeito anticoagulante da dose total de heparina usada durante a CEC conforme o protocolo II, mais dose adicional de $30 \%$ da dose total de protamina. Sendo essa dose iniciada após a dose total. A dose adicional era diluída em $200 \mathrm{ml}$ de soro fisiológico, e administrada em 4 horas na velocidade de infusão de $50 \mathrm{ml} / \mathrm{h}$. em veia periférica.

Terminada a CEC, era feita a neutralização da heparina com cálculo de dose da protamina variando de acordo com os protocolos I e II, porque, até essa fase, o cálculo da dose total de protamina do protocolo III é idêntico ao protocolo II. Foi sempre usada a via aórtica para infusão da protamina. Após dez minutos da neutralização, era realizado o TC de controle para ser comparado com o valor basal e faixa de normalidade. O TC considerado normal foi de 5 a 10 minutos.

Se o resultado do TC fosse normal, era feito um segundo controle 2 horas após a neutralização. Caso o resultado estivesse acima de 10 minutos, era iniciado um esquema de doses de reforço de protamina, na seguinte ordem: primeiro reforço $20 \%$ da dose de protamina administrada no final da CEC, colhendo um TC 10 minutos após. Se o TC continuasse anormal, ou seja, acima de 10 minutos, era administrado um segundo reforço de $10 \%$ da dose de protamina administrada no final da CEC, colhendo um TC 10 minutos após. Caso o TC continuasse anormal, era feito um último reforço de $10 \%$ da dose de protamina administrada no final da CEC, colhendo-se um TC 10 minutos após. Se o TC permanecesse acima de 10 minutos, era tratado como portador de distúrbio da coagulação.

O paciente era avaliado clinicamente com a melhora ou piora do sangramento após cada dose de protamina e só era realizado o fechamento do tórax se considerado em condições hemostáticas dentro dos limites da normalidade.

O protocolo III tinha início a partir da última dose de protamina com TC normal (seja ele medido $10 \mathrm{~min}$ após a protamina, ou após o último reforço), quando era infundida a dose adicional $(30 \%$ da dose total de protamina), na velocidade de $50 \mathrm{ml} / \mathrm{h}$, e colhido TC 2 horas após a última dose de protamina e outro após o termino da dose de infusão contínua. 


\section{RESULTADOS}

A partir desses dados, usamos análise de variância com teste de Bartlet e comparação múltipla de Tukey- Kramer entre os protocolos I, II e III, com o programa GraphPad Start versão 3.0 para Windows 95.

$\mathrm{Na} 1^{\mathrm{a}}$ hora do pós-operatório, os pacientes do protocolo I apresentaram perda sangüínea que variou de 15 a $400 \mathrm{ml}$ com média de $165 \pm 102,7 \mathrm{ml}$; no protocolo II; a perda variou de 0 a $300 \mathrm{ml}$, com média de $116 \pm 74,6 \mathrm{ml} \mathrm{e}$, no protocolo III, a perda variou de 0 a $300 \mathrm{ml}$, com média de $89,6 \pm 73,6 \mathrm{ml}$ (Gráfico 1).

\section{GRÁFICO 1}

\section{PERDA SANGÜÍNEA NA 1a HORA DO PÓS-OPERATÓRIO}

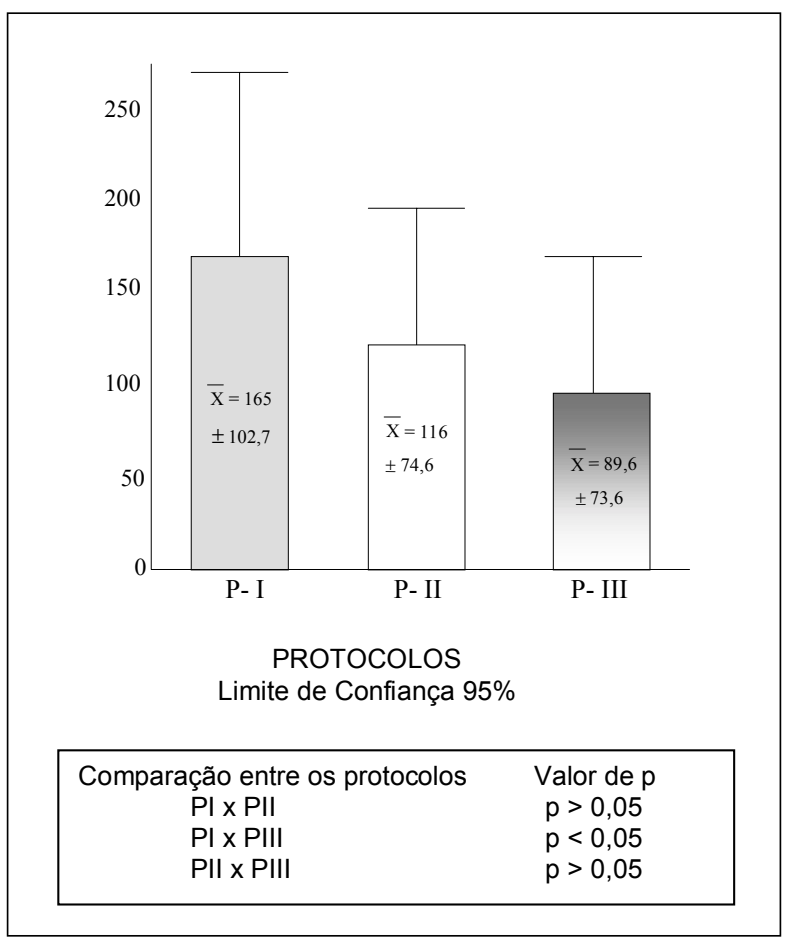

$\mathrm{Na} 12^{\mathrm{a}}$ hora do pós-operatório, os pacientes do protocolo I apresentaram perda sangüínea que variou de 50 a $1220 \mathrm{ml}$, com média de 676,67士 391,4 ml; no protocolo II, a perda sangüínea variou de 100 a $840 \mathrm{ml}$, com média de $423 \pm 236,5 \mathrm{ml}$ e, no protocolo III, a perda sangüínea variou de 100 a $570 \mathrm{ml}$, com média de $329 \pm 213,4 \mathrm{ml}$ (Gráfico 2).

No protocolo I, o volume de sangue ou derivados transfundidos, durante as primeiras 12 horas do pós-operatório, variou de 0 a $7200 \mathrm{ml}$, com média de $1325 \pm 1079,5 \mathrm{ml}$. No protocolo II, o volume transfundido variou de 0 a $2000 \mathrm{ml}$, com média de $660 \pm 599,7 \mathrm{ml}$ e, no protocolo $\mathrm{III}$, o volume transfundido variou de 0 a $1300 \mathrm{ml}$, com média de 486,6 \pm 440,5 ml (Gráfico 3).

\section{GRÁFICO 2}

PERDA SANGÜÍNEA ATÉ $12^{\mathrm{a}}$ HORA DO PÓSOPERATÓRIO

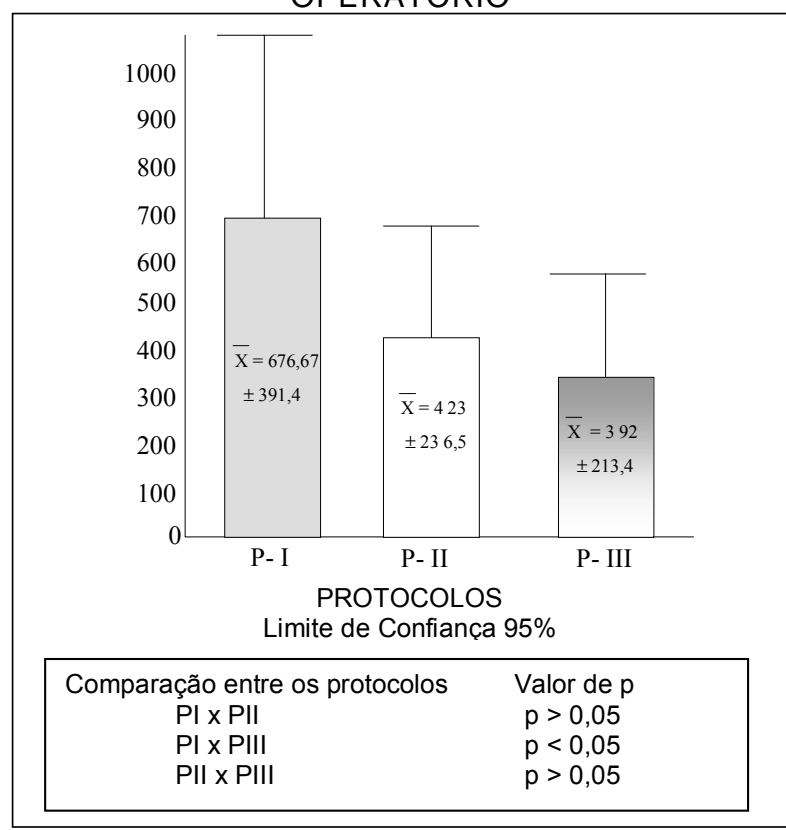

\section{GRÁFICO 3}

VOLUME DE SANGUE OU DERIVADOS SANGÜÍNEOS TRANSFUNDIDOS ATÉ $12^{\mathrm{a}}$ HORA DO PÓS-OPERATÓRIO

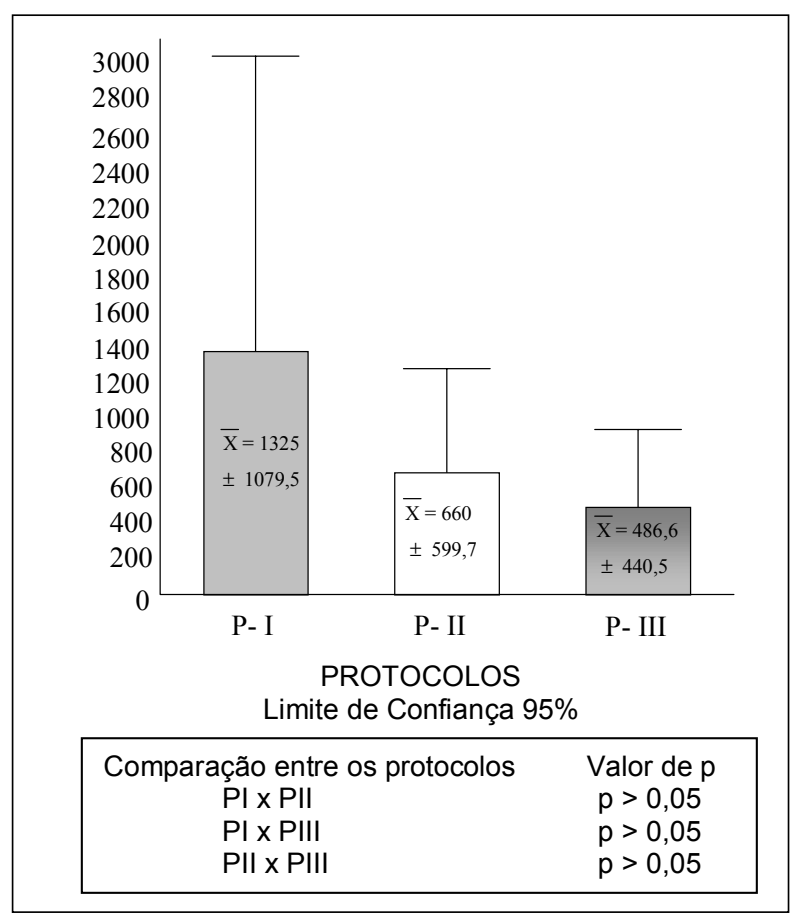


Barroso R C, Mendonça J T, Carvalho M R, Costa R K, Santos J E - Avaliação da protamina na neutralização da heparina após circulação extracorpórea. Rev Bras Cir Cardiovasc 2002; 17(1): 54-60.

Na perda sangüínea da $1^{\underline{a}}$ hora e $12^{\underline{a}}$ hora, houve significância estatística quando analisamos os protocolos I e III. Apesar de não haver significância estatística no volume de sangue transfundido no pós-operatório, quando comparamos os três protocolos, houve uma forte tendência de menor transfusões nos pacientes do protocolo III.

No protocolo I, correram três complicações hemorrágicas, com necessidade de reoperação. Dos pacientes reoperados, 2 apresentaram outras complicações, 1 evoluiu com acidente vascular cerebral (AVC) e outro foi a óbito. Ocorreu 1 caso de AVC sem relação com complicação hemorrágica.

No protocolo II, não houve complicações hemorrágicas nem reoperações. Houve 1 paciente que foi a óbito por infarto agudo do miocárdio (IAM) no pós-operatório imediato, mas sem relação com complicações hemorrágicas.

No protocolo III, não houve complicações hemorrágicas nem reoperações. Um paciente evoluiu com AVC e outro com insuficiência respiratória, sem relação com complicações hemorrágicas.

No protocolo I, $60 \%$ dos pacientes usaram doses de reforço de protamina para controlar o sangramento e corrigir o TC logo na 1a hora do pós-operatório, como também houve a necessidade do uso de sangue para repor volemia e corrigir distúrbio da coagulação.

No protocolo II, 53\% dos pacientes usaram doses de reforço de protamina nas 3 primeiras horas do pós-operatório, porém com menor necessidade de transfusões.

Nenhum paciente necessitou os três reforços de protamina após CEC, conforme preconizava o protocolo.

\section{COMENTÁRIOS}

O maior risco na utilização da heparina durante a CEC não está no excesso de heparina e sim na sua falta, que, por ativar a cascata da coagulação, pode levar ao consumo excessivo das proteínas da coagulação, aumentando os níveis de plasmina, induzindo a fibrinólise e, em alguns casos, levando a coagulação intravascular disseminada. $(1,6,16,17)$.

O TCA tem por finalidade controlar a anticoagulação, para reduzir a dose de heparina; com conseqüente redução da dose de protamina para sua neutralização, mas aumentando o risco de induzir a ativação da cascata da coagulação, principalmente quando o TCA for igual ou inferior a 300 segundos (16). Existem evidências bioquímicas da geração de trombina, indicando ativação do sistema da coagulação e aumento da atividade fibrinolítica, mesmo com TCA acima de 400 segundos ${ }^{(7,8)}$.

BULL et al. (16) afirmam que TCA menor que 180 segundos é inadequado e pode ser fatal, TCA entre 180
- 300 segundos é altamente questionável e TCA superior a 600 segundos é provavelmente desnecessário.

A dose de heparina de $5 \mathrm{mg} / \mathrm{kg}$ com reforços programados é considerada dose elevada, mas nós temos usado esse protocolo desde 1978, com baixos índices de complicações e proporcionando uma anticoagulação segura, sem necessidade de controle periódico do TCA.

As complicações hemorrágicas após a CEC não dependem de um único fator, mas do contato do sangue com superfícies não endotelizadas, trombocitopenia, alteração da função plaquetária, redução das proteínas da coagulação por diluição ou seqüestro, aumento da plasmina circulante por falta de heparina induzindo à fibrinólise e excesso de heparina por neutralização inadequada ou efeito rebote $(1,2,5-7)$.

Foi demonstrado maior tendência de efeito rebote da heparina nos casos em que as doses foram elevadas ${ }^{(17)} \mathrm{e}$, embora haja algum questionamento sobre a re-heparinização retardada, muitos autores acreditam que a heparina, ligada aos tecidos ou endotélio, pode reentrar na circulação; sendo mais provável em pacientes com doença vascular periférica e naqueles submetidos a reaquecimento tardio, com um gradiente de temperatura nasofaríngeo - dedo do pé mantido ${ }^{(3)}$, ou pela presença de protaminases que provoquem a destruição da protamina livre e instabilizem o complexo heparina/protamina com liberação da heparina, restabelecendo a atividade antitrombínica (efeito rebote) (15).

A administração lenta de um acréscimo da dose de protamina nas primeiras horas do pós-operatório pode evitar o efeito rebote, reduzindo a quantidade do sangramento no pós-operatório (1).

Embora o tempo de coagulação, em relação ao TCA, seja um método mais demorado e necessitar de maior controle do laboratório para precisão do resultado, não mudamos nosso protocolo porque alguns estudos mostraram que não houve redução do sangramento ou transfusões no pós-operatório entre os protocolos de dose fixa e os protocolos de doses monitoradas pelo TCA ${ }^{(14)}$; não conseguiram determinar uma dose precisa ou segura para anticoagulação, ao passo que uma monitorização simplificada foi bem sucedida (13) e por não existir consenso sobre um protocolo ideal para administração e neutralização da heparina com protamina para os pacientes submetidos a CEC $(18,19)$.

Em virtude das variações individuais da necessidade de heparina e dos riscos da subdosagem é fundamental a verificação do efeito anticoagulante da dose administrada antes e durante a CEC. A utilização de protocolos padronizados aplicados a todos os pacientes pode ser causa de complicações hemorrágicas, não sendo, por isso, recomendável por alguns autores, devido a que trabalhos demonstram a superioridade da anticoagulação controlada pelo TCA $(2,20,21)$. Por outro lado, JOBES et al. (14) não acharam redução do sangramento ou de trans- 
Barroso R C, Mendonça J T, Carvalho M R, Costa R K, Santos J E - Avaliação da protamina na neutralização da heparina após circulação extracorpórea. Rev Bras Cir Cardiovasc 2002; 17(1): 54-60.

fusões no pós-operatório entre os protocolos de dose fixa e doses monitoradas pela concentração de heparina no sangue, em virtude de o TCA ser prolongado pela hemodiluição, trombocitopenia, alteração da função plaquetária, agentes antifibrinolíticos como aprotinina $(1,2,22,23)$ e hipotermia, independente da concentração de heparina circulante (1,2,7,24-26).

Apesar de usarmos protocolo de doses fixas de heparina, houve menor custo econômico nos protocolos de maior dose de protamina devido ao menor índice de complicações e ao menor número de transfusões. O custo médio no protocolo I foi de $R \$ 14,00$ para heparina e protamina, e de $\mathrm{R} \$ 1600,00$ para o uso de sangue ou seus derivados. No protocolo II, foi de $R \$$ 15,00 para heparina e protamina, e de $R \$ 770,00$ para uso de sangue e seus derivados. No protocolo III, foi de $R \$ 16,00$ para heparina e protamina, e de $R \$ 490,00$ para uso de sangue e seus derivados. Esses valores foram calculados com base no custo hospitalar, no nosso meio, com valor da ampola de heparina igual $R \$$ 2,80 , da ampola de protamina igual $R \$ 1,20$ e de $R \$$ 350 para cada unidade de sangue ou derivados.

A quantidade de protamina para neutralizar a heparina na proporção de 1:1 se mostrou eficaz na sala de operações, por não ser necessário doses de reforço, porém, devido a hipotermia e alterações metabólicas que ocorrem durante a circulação extracorpórea, pode ocorrer, no pós-operatório, liberação lenta, retardada e progressiva da heparina seqüestrada nos tecidos, e sua reentrada na circulação, provocando uma atividade antitrombínica variada, com comprometimento de coagulação e sangramento. Isso foi demonstrado nesse estudo com o protocolo II, onde $53 \%$ dos pacientes necessitaram de doses complementares de protamina nas primeiras horas do pós-operatório.

As doses de reforço de protamina usadas diminuíram o sangramento, mas, como a meia vida da protamina em relação à heparina é menor, dose complementar de protamina se mostrou mais eficaz quando usada em gotejamento durante as primeiras horas do pós-operatório. Isso foi confirmado com o protocolo III, onde nenhum paciente necessitou de reoperação, além de apresentarem menor perda sangüínea e menor volume transfundido.

\section{CONCLUSÕES}

Concluímos que: 1 - A dose de protamina para neutralizar a heparina na proporção menor que 1:1 tem maior necessidade de doses de reforço de protamina no pós-operatório, maior necessidade de transfusões e mais complicações hemorrágicas. 2 - A neutralização da heparina com dose de protamina na proporção de 1:1 não é totalmente eficaz, devido à maioria dos pacientes (53\%) necessitarem de doses complementares nas primeiras horas do pós-operatório. 3 - Dose adicional de $30 \%$ da dose total de protamina na proporção de 1:1 nas primeiras horas do pós-operatório reduz a perda sangüínea e a necessidade de transfusões.

RBCCV 44205-572

Barroso R C, Mendonça J T, Carvalho M R, Costa R K, Santos J E - Evaluation of the protamine in the neutralization of heparin after cardiopulmonary bypass. Rev Bras Cir Cardiovasc 2002; 17(1): 54-60.

ABSTRACT: Introduction: The protamine is the antidote universally used to neutralize the heparin at the end of the cardiopulmonary bypass; however there isn't a consensus about the ideal necessary dosage so far.

Objective: To evaluate the effectiveness of the neutralization of heparin, with variable dosages of protamine after cardiopulmonary bypass, using three different protocols.

Material and Methods: From April to August 2000, 45 patients were randomized prospectively, and divided into three Protocols: I, II e III with 15 patients each. The protocol I evaluates the neutralization of heparin, with dosage of protamine calculated by the initial dosage of heparin. The protocol II evaluates the neutralization of the full dosage of heparin used during cardiopulmonary bypass. The protocol III evaluates the neutrlization of the full dosage of heparin, plus a reinforcement of $30 \%$ of this dosage, administered in peripheral vein during four hours.

Results: In the protocol I, $60 \%$ of the patients had necessity of reinforcement of the dosage of protamine and $20 \%$ showed hemorrhagics complications, requiring reoperation. From these $20 \%$, one patient passed away and another had cerebrovascular accident; $53 \%$ of the patients of the protocol II had necessity of reinforcement of the dosage of protamine. The patients from protocols II and III didn't have neither hemorrhagics complications nor necessity of reoperation.

Conclusions: 1 - The dosage of protamine to neutralize heparin in the proportion less than 1:1, isn't sufficient. The patients that need of doses of reinforcement, have more hemorrhagics complications and greater necessity of blood transfusions. 2 - The neutralization of heparin, with dosage of protamine in the proportion of $1: 1$ isn't totaly effective; $53 \%$ of the pacients needed complementary doses in the frist hours affter surgery. 3 - An additional dose of $30 \%$ in the dosage of protame in the proportion of $1: 1$ in continual infuse in the frist hours after surgery, decreases the loss of blood and the necessity of transfusions.

DESCRIPTORS: Protamines, administration and dosage. Heparin, adverse effects. Extracorporeal circulation. 
Barroso R C, Mendonça J T, Carvalho M R, Costa R K, Santos J E - Avaliação da protamina na neutralização da heparina após circulação extracorpórea. Rev Bras Cir Cardiovasc 2002; 17(1): 54-60.

\section{REFERÊNCIAS BIBLIOGRÁFICAS}

1 Souza M H L \& Elias D O - Fundamentos da circulação extracorpórea. Vol. I. Rio de Janeiro: Centro Editorial Alfa Rio, 1995: 255-73.

2 Despotis G J, Gravlee G, Filos K, Levy J, Fisher D M - Anticoagulation monitoring during cardiac surgery. Anesthesiology 1999; 91: 1122-51.

3 Spiess B D \& Chang S PB - Distúrbios peroperatórios da coagulação. In: Thomas S J \& Kramer JL, eds. Manual de anestesia cardíaca. 2 ed. Rio de Janeiro, Revinter, 2000: 492-525.

4 Liu B, Belboul A, Larsson S, Roberts D - Factors influencing haemostasis and blood transfusion in cardiac surgery. Perfusion 1996; 11:131-43.

5 Tabuchi N, de Haan J, Boonstra P W, van Oeveren W - Activation of fibrinolysis in the pericardial cavity during cardiopulmonary bypass. J Thorac Cardiovasc Surg 1993; 106: 828 - 33.

6 Gravlee G P, Rogers A T, Dudas L M et al. - Heparin management protocol for cardiopulmonary by pass influences postoperative heparin rebound but not bleeding. Anesthesiology 1992; 76: 393 -401.

7 Gravlee G P, Haddon W S, Rothberger H K et al. - Heparin dosing and monitoring for cardiopulmonary bypass: a comparison of techniques with measurement of subclinical plasma coagulation. J Thorac Cardiovasc Surg 1990; 99: 518 -27.

8 Young J A, Kister C T, Doty D B - Adequate anticoagulation during cardiopulmonary bypass determined by activated clotting time and the appearance of fibrin monomer. Ann Thorac Surg 1978; 26: $231-40$.

9 Katz N M, Kim Y D, Siegelman R, Ved S A, Ahmed S W, Wallace R B - Hemodinamics of protamine administration comparison of right atrial, left atrial, and aortic injections. J Thorac Cardiovasc Surg 1987; 94: 881-6.

10 Weiler J M, Freiman P, Sharath MD et al. - Serious adverse reactions to protamine sulfate: are alter natives needed? J Allergy Clin Immunol 1985, 75: 297-303.

11 Perkash A - A comparision of the quantitative action of protamine and heparin on blood coagulation significance in clinical and laboratory usage. $A m \mathrm{~J}$ Clin Pathol 1980; 73: 767-81.

12 Miyashita T, Nakajima T, Hayashi Y, Kuro M - Hemostatic effects of low-dose protamine following cardiopulmonary bypass. Am J Hematol 2000; 64:112-5.

13 Bull B S, Korpman R A, Hurse W M et al. - Heparin therapy during extracorporeal circulation: I. Problems inherent in existing heparin protocols. $J$ Thorac Cardiovasc Surg 1975; 69: 674-84.
14 Jobes D R, Scwartz A J, Ellison N, Andrews $\mathrm{R}$ Ruffini R A, Ruffini J J - Monitoring heparin anticoagulation and its neutralization. Ann Thorac Surg 1981; 31: 161-6.

15 Shanberge J N, Murato M, Quattrociocchi-Longe T, van Neste L - Heparin- protamine complexes in the production of heparin rebound and other complications of extracorporeal bypass procedures. Am J Clin Pathol 1987; 87: 210-7.

16 Bull B S, Huse W M, Braner F S, Korpman R A- Heparin therapy during extracorporeal circulation. II. The use of dose response curve to individualize heparin and protamine dosage. J Thorac Cardiovasc Surg 1975; 69: $685-9$

17 Kalter R D, Saul C M, Wetstein L, Soriano C, Reiss R F - Cardiopulmonary bypass: associated hemostatic abnormalities. J Thorac Cardiovasc Surg 1979; 77: 427-35.

18 Akl B F, Vargas G M, Neal J, Robillard J, Kelly PClinical experience with the activated clotting time for the control of heparin and protamine therapy during cardiopulmonary bypass. J Thorac Cardiovasc Surg 1980; 79: 97-102.

19 Esposito R A, Culliford A T, Colvin S B, Thomas S J, Lackner $\mathrm{H}$, Spencer $\mathrm{F} \mathrm{C} \mathrm{-} \mathrm{The} \mathrm{role} \mathrm{of} \mathrm{the} \mathrm{activeted}$ clotting time in heparin administration and neutralization for cardiopulmonary. J Thorac Cardiovasc Surg 1983; 85: 174-85.

20 Dearing J P, Bartles D M, Stroud M R, Sade R MActivated clotting times versus protocol anticoagulation management. J Extracorporeal Technol 1983:15:17-9.

21 Lefemine A A \& Lewis M - Activated clotting time for control of anticoagulation during surgery. Ann Surg 1985; 51: 274-8.

Schaadt $\mathrm{J}$-Monitoring anticoagulation during aprotinin utilization. J Extracorporeal Technol 1996; 28: 42-7.

23 Kase P B, Dearing J P - Factors affecting the activated clofting time. J Extracorporeal Technol 1985; 17: 27-30.

24 Aren C, Feddersen K, Radegran K - Comparison of two protocols for heparin neutralization by protamine after cardiopulmonary bypass. J Thorac Cardiovasc Surg 1987; 94: 539-41.

25 Shirota K, Watanabe T, Takagi Y, Ohara Y, Usui A, Yasuura $\mathrm{K}$ - Maintenance of blood heparin concentration rather than activated clotting time better preserves the coagulation system in hypothermic cardiopulmonary bypass. Artif Organs 2000; 24: 49-56.

26 Leyvi G, Shore-Lesserson L, Harrington D, Vela-Cantos F, Hossain S - An investigation of a new activated clotting time "MAX - ACT" in patients undergoing extracorporeal circulation. Anesth Analg 2001; 92: 578 - 83. 